\title{
Selection related to musculoskeletal complaints among employees
}

Bart C H de Zwart, Jake P J Broersen, Allard J van der Beek, Monique $\mathrm{H}$ W Frings-Dresen, Frank J H van Dijk

\begin{abstract}
Objectives-To (a) describe differences in the outcome of cross sectional and longitudinal analysis on musculoskeletal complaints relative to age and work demands, and $(b)$ to assess the entrance and drop out selection on musculoskeletal complaints among groups of employees relative to age and work demands.

Methods-A study population was selected on the basis of questionnaire data from periodical occupational health surveys of almost 45000 employees collected between 1982 and 1993. From all companies within this data base that participated twice in company wide surveys four years apart, male employees were selected, and stratified for age and work demands. There were several populations: follow up (participation in both surveys); drop out (participation only in the first survey); entrance (participation only at the second survey); and two cross sectional populations (all participants at each survey). Prevalences of back complaints and turnover rates were analysed.
\end{abstract}

Results-Reported back complaints in the cross sectional analysis declined over the oldest age groups in heavy physical work versus a small increase in the longitudinal analysis. The age group 50-9 and back complaints were identified as predictors at the first survey for not participating at the second survey. Neither age nor work demands at the first survey indicated drop out among those employees with back complaints at the first survey. The effects of entrance selection on estimated prevalences were small.

Conclusions-The results indicate that musculoskeletal disorders lead to selection out of work, affecting the validity of both cross sectional and longitudinal epidemiological studies. In future studies analyses of turnover figures on musculoskeletal complaints relative to work demands and age are recommended.

\section{(Occup Environ Med 1997;54:800-806)}

Keywords: back complaints; age; work demands; selection; study design; drop out; entrance

During the past few years, various studies have been carried out on the combined effects of physical work demands and age on the development of musculoskeletal complaints, disorders, and related work incapacity. The two principle methods applied in these studies are the cross sectional ${ }^{1-5}$ and longitudinal design. ${ }^{67}$ When comparing the outcome of these research studies, remarkable differences in results are found between the types of methodological designs.

It is obvious that prevalences and incidences obtained either by cross sectional or by longitudinal studies must be interpreted with caution because of a health related selection effect. ${ }^{8}$ Essentially, two types of health related selection mechanisms are relevant in most occupational cohorts: entrance and drop out selection. ${ }^{10}$ Health related entrance selection refers to the selection of healthy members from the general population during the process of hire. For example, people with musculoskeletal impairments or with a low physical work capacity probably will not choose a strenuous job for themselves (self selection), or are not selected by a personnel officer on the outcome of a pre-employment medical examination. Health related drop out selection, on the other hand, refers to the process of employees leaving their occupation, involving people with a relatively low or diminished work capacity, possibly as a result of health problems. For example, employees in strenuous physically demanding jobs who have chronic musculoskeletal complaints may decide to choose a less strenuous job or, if they continue their work, become disabled for their job. Consequently, this health related selection in occupational cohorts yields a bias that tends to mask real occupational health risks in cross sectional as well as longitudinal studies. ${ }^{8}$

In general, it is suggested that this health based selection is subject to substantial modification by factors such as age and work demands. ${ }^{11}$ For example, it may be hypothesised that the effects of entrance selection on the outcome of musculoskeletal complaints at group level are more pronounced in younger than older employees in physically demanding work. The obvious reason is that the number of elderly people starting to work for the first time in a heavy physical job is small. On the other hand, the effects of drop out selection are expected to be more pronounced in the older age groups, as the risk of developing musculoskeletal complaints increases with age. ${ }^{7}$ Furthermore, type of work demands may also be a crucial factor in this matter. For most physically demanding occupations, an optimal condition of the musculoskeletal system is a prerequisite. Consequently, entrance and out- 
Population

Follow up (heavy physical (Fp)/mental (Fm)):

Participating in first survey (T1) and four years later in second survey (T2), and at T1 and T2 working in the same company in a heavy physically demanding/mentally demanding job

Drop out (heavy physical (Dp)/mental (Dm)):

Participating in survey at $\mathrm{T} 1$ working in a heavy physically demanding/mentally demanding job, and not participating at $\mathrm{T} 2$, or participating at T2 while working in a different company compared with $\mathrm{T} 1$, and/or job at T2 not categorised as heavy

physically demanding/mentally demanding

Entrance (heavy physical (Ep)/mental (Em)):

Participating in survey at T2 working in a heavy physically demanding/mentally demanding job, and not participating at T1, or participating at $\mathrm{T} 1$ while working in a different company compared with $\mathrm{T} 2$, and/or job at $\mathrm{T} 1$ not categorised as heavy physically demanding/mentally demanding

Cross sectional (heavy physical $(\mathrm{Cp}) /$ mental $(\mathrm{Cm})$ ):

At T1: CpT1/CmT1 is a composite of the populations FpT1/FmT1 and Dp/Dm

At T2: CpT2/CmT2 is a composite of the populations FpT2/FmT2 and Ep/Em

flow selection related to musculoskeletal complaints may be expected to be more pronounced within physically demanding than with sedentary occupations.

Coming back to our initial point, it may be hypothesised that both types of selection may have different effects in cross sectional and longitudinal study designs. This may be a plausible explanation for reported differences in study results between the two designs. Hence, to prevent drawing false conclusions from these studies, knowledge about the selection mechanism present within the population under study is required. However, the effects of health related selection relative to age and work demands on the outcome of cross sectional and longitudinal studies have been poorly defined so far.

The purpose of this study is twofold: $(a)$ to describe differences in the outcome of cross sectional and longitudinal analysis on musculoskeletal complaints relative to age and work demands, and $(b)$ to assess the entrance and drop out selection on musculoskeletal complaints among groups of employees relative to age and work demands.

\section{Materials and methods}

STUDY DESIGN

In The Netherlands, regional occupational health services take care of the health and wellbeing of employees of affiliated companies. With intervals of around four years, all employees are invited by their regional occupa- tional health services to participate in a periodic occupational health survey. ${ }^{72}$ Within the interval 1982 to 1993 , almost 45000 employees, working in a wide range of occupations within 354 companies, participated in at least one survey carried out by an occupational health service in the eastern part of The Netherlands. From this population, only male employees were selected from companies participating at least twice in a company wide survey in this period. The interval between the first and second survey period within each company was restricted to between 2.5 and 5.5 years, mean (SD) $3.7(0.4)$ years. When a company participated more than twice, the first two survey periods were selected.

From the selected companies, all employees participating in at least one out of two survey periods were stratified for work demands. Stratification was based on employee's job title according to the classification described by $\mathrm{De}$ Zwart et $a l^{137}$ For the present study, only employees within the two most contrasting categories were selected-that is, employees in the categories mentally demanding work and heavy physically demanding work. These categories will be referred to as mental and heavy physical.

In total, survey questionnaire data of male employees from 188 different companies were selected. On the basis of characteristics of participation in a survey and other criteria (table 1), employees were classified into several populations: a cross sectional population at the

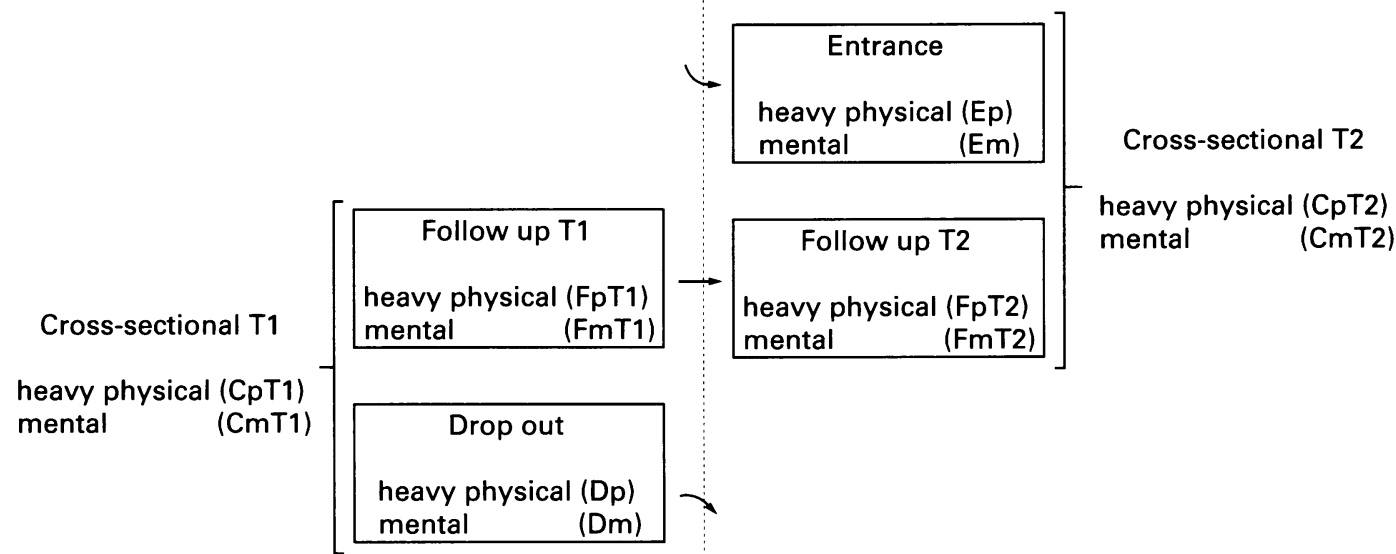

Figure 1 Study populations at the first survey (T1) and four years later at the second survey (T2). 

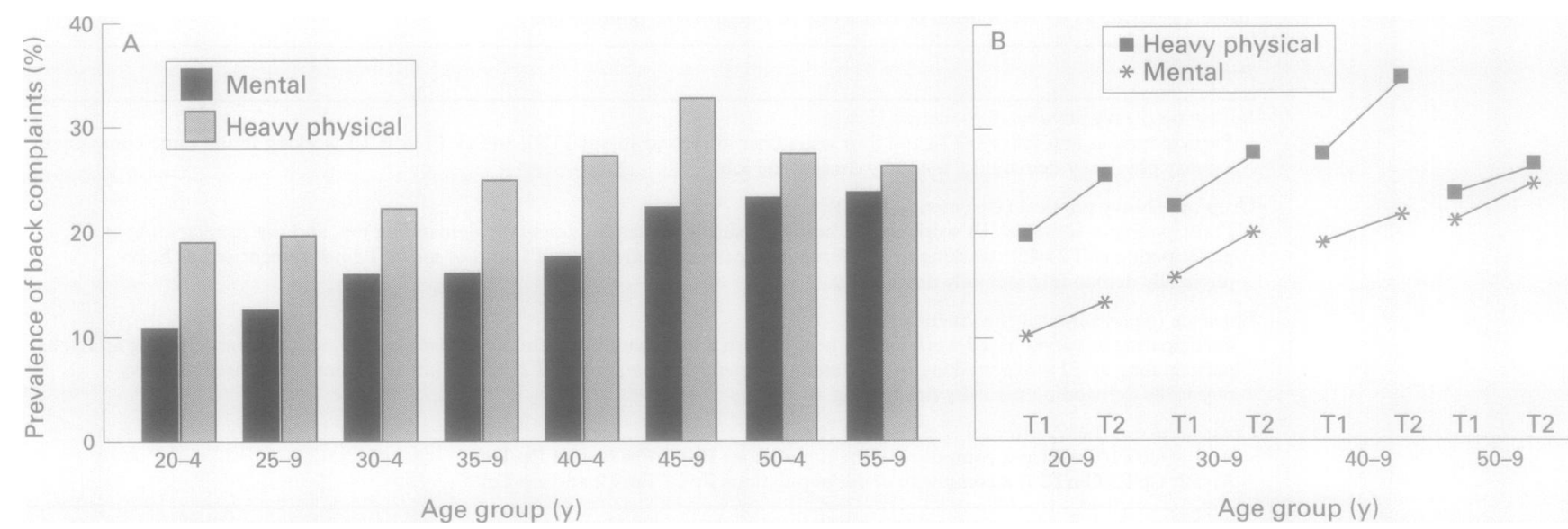

Figure 2 Prevalence of back complaints by age group and types of work demands based on cross sectional analyses at (A) the first survey (T1) of the populations $C p T 1(n=5683)$ and $C m T 1$ ( $n=4423)$ and $(B)$ four year follow up analyses of populations $F p(n=3858)$ and $F m(n=3080)$. Follow up age groups are classified according to age at the first survey.

first survey consisting of a follow up population (participating in both surveys), a drop out population (participating at the first survey only), a cross sectional population at the second survey consisting of a follow up population (those who also participated at the first survey) and an entrance population (participating at the second survey only). Figure 1 shows the various populations under study.

\section{DATA ANALYSIS}

Back complaints are widely recognised as one of the most common musculoskeletal complaints affecting employees. ${ }^{14}$ Therefore, data on the question "Do you regularly have pain or stiffness in the back? yes/no", originating from the survey questionnaire, were used for analysis. For the different populations, prevalences were computed. All populations were stratified into four age groups $(20-9,30-9,40-9$, and 50-9). Figure 2 A shows the cross sectional results at the first survey; eight age groups were constructed covering five year intervals each to analyse the differences in results between both types of study design more precisely.

Multiple logistic regression analysis was applied to test whether the determined collection of variables could be identified as predictors at the time of the first survey for drop out at the second survey. Drop out at the second survey was included in the model as a dichotomous outcome variable. Age at the first survey was included as a categorical scaled independent variable. Type of work demands and back complaints at the first survey were included as dichotomous independent variables. All three independent variables were forced in a single step into the model. The measure of association was the odds ratio. ${ }^{15}$ Furthermore, interaction terms were entered into the model in a second step by forward stepwise selection (cut off value of $P<0.05$ ).

Differences in prevalences between the different populations were tested for significance by a two by two table $\chi^{2}$ test. Within the follow up population, differences in prevalence between the first and the second survey were tested by means of the non-parametric $\mathrm{McNe}-$ mar test for two correlated dichotomous variables. Differences were considered significant when the $P$ value was $<0.05$.

\section{Results}

CROSS SECTIONAL VERSUS LONGITUDINAL ANALYSIS

Figure 2 shows the prevalences of back complaints by age and work demands based on cross sectional analyses (A) and follow up analyses (B). Within both designs, there was a trend for higher prevalences among employees in heavy physical work than those in mental

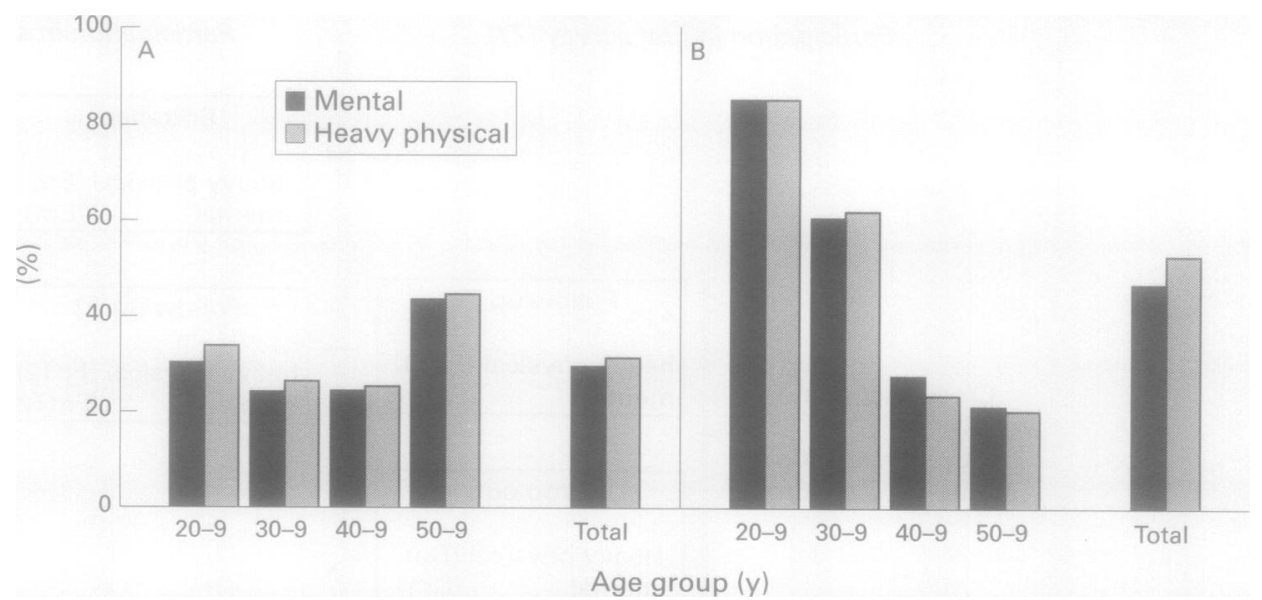

Figure 3 (A) Percentage of drop out in total population at the first survey (T1) and (B) percentage of entrance in the total population at the second survey (T2), stratified by age and types of work. 
Table 2 Prevalences (PRs) of back complaints and number of male employees ( $n$ ) in the follow up, drop out, and total cross sectional population at the first survey (T1), stratified for age and types of work demands (age group refers to age at T1)

\begin{tabular}{|c|c|c|c|c|c|c|c|c|}
\hline \multirow[b]{3}{*}{ Population at $T 1$} & \multicolumn{8}{|c|}{ Age group (y) } \\
\hline & \multicolumn{2}{|l|}{$20-9$} & \multicolumn{2}{|l|}{$30-9$} & \multicolumn{2}{|l|}{$40-9$} & \multicolumn{2}{|l|}{$50-9$} \\
\hline & $P R(\%)$ & $n$ & $P R(\%)$ & $n$ & $P R(\%)$ & $n$ & $P R(\%)$ & $n$ \\
\hline \multicolumn{9}{|l|}{ Heavy physical: } \\
\hline Follow up (FpT1) & 19.9 & 614 & 22.7 & 1146 & 27.6 & 1323 & 23.9 & 775 \\
\hline Drop out (Dp) & 19.4 & 320 & $27.7^{\star}$ & 423 & $36.8^{\star \star \star}$ & 456 & $30.7^{\star \star}$ & 626 \\
\hline Cross sectional (CpT1) & 19.7 & 934 & 24.0 & 1569 & 30.0 & 1779 & 26.9 & 1401 \\
\hline \multicolumn{9}{|l|}{ Mental: } \\
\hline Follow up (FmT1) & 10.1 & 336 & 15.8 & 1076 & 19.0 & 1074 & 21.2 & 594 \\
\hline Drop out (Dm) & $16.6^{\star}$ & 151 & 16.8 & 363 & 22.3 & 354 & 26.1 & 475 \\
\hline Cross sectional (CmT1) & 12.1 & 487 & 16.1 & 1439 & 19.8 & 1428 & 23.4 & 1069 \\
\hline
\end{tabular}

${ }^{\star} \mathrm{P}<0.05 ;{ }^{\star \star} \mathrm{P}<0.01 ;{ }^{\star \star \star} \mathrm{P}<0.001, \chi^{2} \mathrm{PR}$ at follow up $v \mathrm{PR}$ in drop out population within same age group.

Table 3 Multivariate predictors at T1 for drop out four years later at $T 2$

\begin{tabular}{lll}
\hline Predictor & Odds ratios & $(95 \%$ CI) \\
\hline Age group at T1 (y): & 1.00 & Reference \\
$20-9$ & 0.71 & $(0.62$ to 0.82$)$ \\
$30-9$ & 0.67 & $(0.58$ to 0.77$)$ \\
$40-9$ & 1.59 & $(1.39$ to 1.83$)$ \\
$50-9$ & 1.00 & Reference \\
Work demands at T1: & & $(0.96$ to 1.14$)$ \\
Mental & 1.04 & \\
$\quad$ Heavy physical & & Reference \\
Back complaints at T1: & 1.00 & $(1.18$ to 1.45$)$ \\
$\quad$ No complaints & 1.31 & \\
Complaints & & \\
\hline
\end{tabular}

work in all age groups. Within the cross sectional design, a rise in complaints was reported up to the group aged 45-9 in heavy physical work with a significant difference in prevalences between the successive age groups $40-4$ and 45-9 $(P<0.05)$. After this age group, a significant decline in prevalence was found from $33 \%$ to $28 \%$ in the age group $50-4$ years $(\mathrm{P}<0.05)$ ending at $26 \%$ in the group aged 55-9. In mental work there was a rising trend up to the age group 45-9, with a significant difference only present between the successive age groups $40-4$ and $45-9(P<0.05)$. After these age groups only a small increase was found in the two oldest age groups. Within the follow up analyses, for all age groups in heavy physical work and mental work an increase was reported in prevalence of back complaints over the four year interval (fig 2 B). In heavy physical work these increases were significant in the youngest age group $(P<0.01)$ and both middle aged groups $(\mathrm{P}<0.001)$. A high increase from $28 \%$ to $35 \%$ was reported in the age group 40-9. For mental work, significant increases were reported for the groups aged 30-9 $(P<0.001)$ and $40-9(P<0.05)$.

\section{DROP OUT POPULATION}

Of all employees in heavy physical work participating in the first survey, $32 \%$ did not participate in the second survey four years later (fig 3). In mental work, a nearly equal drop out percentage of $30 \%$ was reported. For both types of work, the highest drop out was in the oldest age groups (around $45 \%$ of all participants at the first survey) followed by the youngest age group (around 32\%).

Table 2 presents prevalences of back complaints for the different populations at the first survey. In all age groups in heavy physical work, with the exception of the youngest one, significantly higher prevalences of back complaints occurred for employees in the drop out population $(\mathrm{Dp})$ than in those in the follow up population (FpT1). For the employees aged 40-9, the prevalence of back complaints in the drop out population was $9 \%$ higher than in the follow up population $(\mathrm{P}<0.001)$. Also in mental work, higher prevalences among employees who dropped out occurred, with a significant difference in prevalence of nearly $7 \%(P<0.05)$ in the youngest age group.

Table 3 shows the results of multiple logistic regression analysis, which show that the age 50-9 at the first survey was the strongest predictor for drop out at the second survey compared with the control age group 20-9 years (odds ratio $1.59,95 \%$ confidence interval (95\% CI) 1.39 to 1.83 ). Back complaint at the first survey was also identified as a predictor for drop out at the second survey with an odds ratio of 1.31 (95\% CI 1.18 to 1.45$)$. Neither

Table 4 Prevalences (PRs) of back complaints and number of male employees ( $n$ ) in the follow up, entrance, and total cross sectional population at the second survey (T2), stratified for age and types of work demands (age group refers to age at T2)

\begin{tabular}{|c|c|c|c|c|c|c|c|c|}
\hline \multirow[b]{3}{*}{ Population at $T 2$} & \multicolumn{8}{|c|}{ Age group (y) } \\
\hline & \multicolumn{2}{|l|}{$20-9$} & \multicolumn{2}{|l|}{$30-9$} & \multicolumn{2}{|l|}{$40-9$} & \multicolumn{2}{|l|}{$50-9$} \\
\hline & $P R(\%)$ & $n$ & $P R(\%)$ & $n$ & $P R(\%)$ & $n$ & $P R(\%)$ & $n$ \\
\hline \multicolumn{9}{|l|}{ Heavy physical: } \\
\hline Follow up (FpT2) & 23.3 & 348 & 26.7 & 858 & 32.9 & 1463 & 30.3 & 1124 \\
\hline Entrance (Ep) & $18.1^{\star}$ & 2025 & 26.7 & 1415 & 31.7 & 464 & 30.1 & 306 \\
\hline Cross sectional (CpT2) & 18.9 & 2373 & 26.7 & 2273 & 32.6 & 1927 & 30.3 & 1430 \\
\hline \multicolumn{9}{|l|}{ Mental: } \\
\hline Follow up (FmT2) & 9.0 & 134 & 19.1 & 754 & 20.9 & 1201 & 23.8 & 905 \\
\hline Entrance (Em) & 10.2 & 783 & 14.9 & 1171 & 24.4 & 472 & 23.8 & 261 \\
\hline Cross sectional (CmT2) & 10.0 & 917 & 16.5 & 1925 & 21.9 & 1673 & 23.8 & 1166 \\
\hline
\end{tabular}

${ }^{\star} \mathrm{P}<0.05 ;{ }^{\star \star} \mathrm{P}<0.01 ;{ }^{\star \star \star} \mathrm{P}<0.001, \chi^{2} \mathrm{PR}$ at follow up $v \mathrm{PR}$ in entrance population within same age group. 
work demands at the first survey nor the various interaction terms entered in step two were found to contribute to the logistic regression model.

ENTRANCE POPULATION

About $50 \%$ of all participants in the second survey were in the entrance population (fig 3), with large differences between the age groups. A steep and almost identical decline in the entrance population for both types of work was found with increasing age, starting from about $85 \%$ in the youngest age group to about $20 \%$ in the oldest age group.

Table 4 presents the prevalences of back complaints for the populations at the second survey. The entrance population in heavy physical work (Ep) had a significantly lower prevalence than the follow up group at the second survey (FpT2) in the youngest age group $(P<0.05)$. In mental work this was also found for the group aged 30-9 (P<0.05).

\section{Discussion}

STUDY POPULATION

Survey questionnaire data gathered between 1982 and 1993 were used for secondary analysis. For the employees in the drop out population as well as the entrance population, the actual reasons for not participating twice in a company wide survey were not registered. Van Veldhoven et $a l^{16}$ studied these reasons in more detail in a similar setting. From all drop outs in their survey, $80 \%$ of the employees were no longer doing the same job at the second survey (mean follow up time 3.5 years). This may be attributed to a change in job, disablement, retirement, dismissal, death, etc. The remaining $20 \%$ were classified as non-responders at the second survey. Among the entrance group at the second survey in their study, $88 \%$ of the employees were not doing the same job during the first survey and $12 \%$ were non-responders at the first survey. From these numbers it can be assumed that by far the main reason for not participating twice in our study was related to leaving the job or the company and not to nonresponse.

\section{DROP OUT POPULATION}

Age was found to be an important factor associated with drop out, especially for those employees aged 50-9. Within this age group, the main reasons for a high drop out may be related to disability and early retirement. ${ }^{17}$ However, the youngest age group (20-9) also had a relatively large drop out percentage. Although in the scientific literature health related factors such as musculoskeletal complaints and cardiovascular diseases are often reported as the most predominant reasons for elderly employees to leave their job, ${ }^{18}$ for the younger ones non-health related factors such as dissatisfaction with the salary or irregular working hours often seem to be related to drop out. ${ }^{19} 20$

In heavy physical work, in all but the youngest age group significantly higher prevalences of back complaints occurred in the drop out population than the follow up population. The absence of significant differences within the youngest age group may be explained by the small number of years in which younger workers have been generally exposed to strenuous work demands. According to this line of reasoning, the risk of developing serious musculoskeletal complaints leading to drop out within this short exposure period can be expected to be low. In this study, the presence of back complaints at the first survey was found to increase the probability of not participating in the second survey four years later. From this result, it may be assumed that back complaints are an important determinant of health related turnover. When related to musculoskeletal complaints in general, this finding accords with the results of various studies. ${ }^{182021}$

Surprisingly, an interaction effect between age and back complaints at the first survey and drop out at the second survey was absent. This suggests that the higher probability of drop out when back complaints are present does not differ between the various age groups. Furthermore, type of work does not affect the relation between back complaints at the first survey and drop out at the second survey. Hence, although heavy physical work demands often have been identified as a risk factor for the development of back complaints, ${ }^{22}$ they were not associated with an increased probability of drop out among employees with these back complaints.

\section{ENTRANCE POPULATION}

Within the present study, only for the youngest age group was there a significantly lower prevalence of back complaints in the entrance population into heavy physical work than in the follow up group at the second survey. For the older age groups, perhaps those employees in the entrance group were also exposed to harmful physical work demands in their earlier working life. This might explain the absence of significant differences in prevalences.

Analysing the entrance percentages stratified for age, it is not surprising that the process of entering an occupation is typically related to the younger age groups within both types of work demands. With increasing age the number of people starting a new job rapidly declines. The overall entrance in the total population at the second survey was found to be quite high, about $50 \%$. Van Veldhoven et $a l^{16}$ reported an entrance percentage of about $40 \%$. The high entrance percentage in our study can partly be explained by expansion of some of the companies during the follow up interval.

SELECTION BIAS AND METHODOLOGICAL DESIGN Health related selection in occupational cohorts has been found to bias estimated morbidity rates to a considerable degree, severely affecting the validity of cross sectional and longitudinal epidemiological studies. ${ }^{8923}$ Within our study, the most striking difference between the cross sectional and longitudinal design was reported in the oldest age groups (50-9). These controversial results might partly be ascribed to the effects of health related selection. The data of our study showed that health related selection due to back 
complaints is not only present among elderly employees, but also among the younger ones. The cumulative effect of the selection process over all age groups finally results in a relatively healthy survivor population in elderly employees. This might explain why prevalences of musculoskeletal complaints normally flatten or even decline with increasing age in cross sectional analyses, as reported in our study and in others. ${ }^{3524}$

The apparent difference in the trend in cross sectional assessed prevalences in the older age groups as found between employees in heavy physical work (decline) and those in mental work (stable) is thought partly to be the result of health related selection. Although an effect of work demands on the relation between back complaints and drop out was absent, in all age groups the prevalence of back complaints was higher among employees in heavy physical work than in mental work. Consequently, a relatively similar number of drop outs among those employees with complaints in both types of work leads to a limited increase or even a decline in prevalence in heavy physical work compared with mental work. This will be found particularly when the greater number of drop outs with complaints in heavy physical work is not combined with a greater number of employees developing new complaints. The results of our previous study showed that only in the older age groups a relation between type of work and the risk of developing back complaints was absent. ${ }^{7}$ However, judging from our data, it is not the only reason for the differences in the relations between age, complaints, and type of work. Other explanations could be more efficient working methods used by older employees in heavy physical work or a redistribution of physically heavy tasks from the older to the younger employees, ${ }^{25}$ reducing the risk of musculoskeletal complaints in the older group.

Bias from health related drop out during the follow up interval is considered to be one of the major problems in longitudinal occupational epidemiological studies. $^{8}$ Here, the strong decline in prevalence of complaints at the first survey between the two oldest age groups in heavy physical work only may also partly be attributed to the selection effects described already for the cross sectional analysis. A reasonable explanation for the small increase in prevalence between the first survey and the second survey in the oldest age group in heavy physical work when compared with the other age groups is the smaller risk of developing back complaints within this healthy survivor population of elderly employees. ${ }^{\text {? }}$

Contrary to our expectations, differential effects of type of work on selection based on back complaints were not found. Thus our results could not support the theory that in general, differences in selection bias between the population exposed to heavy physical work versus the control population significantly mask work related musculoskeletal disorders in epidemiological studies. ${ }^{8}$

Finally, considering the effects of health related selection as discussed in this paper, the question arises whether cross sectional and longitudinal designs can be accurate methods for studying the combined effects of physical work demands and age on the development of musculoskeletal complaints. Both designs seem to have their own advantages and disadvantages. Well designed longitudinal studies, however, seem to be more informative for studying the relation between long lasting exposure to physical workload and adverse health effects in combination with the aging process. However, based on our results we would strongly recommend that, if possible, the target for examination in future studies should not be restricted to those who stay within the occupational follow up cohort only, but should also include analyses of health status and work characteristics of those who leave their job. The analysis of turnover figures may serve as an additional or even essential strategy for estimating the risk of developing work related musculoskeletal complaints. This approach, unfortunately seldom applied in ergonomic epidemiological studies, can provide crucial information needed to correctly identify ergonomic hazards in the work situation. ${ }^{26}$

We thank the staff of the Occupational Health Service "Oost-Gelderland" in Doetinchem for providing their data on periodical occupational health survey.

1 Törner M, Blide G, Eriksson H, Kadefors R, Karlsson R, Petersen I. Musculo-skeletal symptoms as related to workPetersen $\mathrm{I}$. Musculo-skeletal symptoms as related to working conditions among Swedish profe

2 Applied Ergonomier M, Laurig W, Schlegel KF. Orthopaedic Luttmann A, Jäger M, Laurig W, Schlegel KF. Orthopaedic
diseases among transport workers. Int Arch Occup Environ diseases among transport

3 Broersen JPJ, De Zwart BCH, Van Dijk FJH, Meijman TF Van Veldhoven $M$. Health complaints and workin conditions experienced in relation to work and age. Occup Environ Med 1996;53:51-7.

4 Liira JP, Shannon HS, Chambers LW, Haines TA Long-term back problems and physical work exposures in the 1990 Ontario Health Survey. Am $\mathcal{F}$ Public Health 1996; 86:382-387.

5 De Zwart BCH, Broersen JPJ, Frings-Dresen MHW, Van Dijk FJH. Musculoskeletal complaints in the Netherlands in relation to age, gender, and physically demanding work. Int Arch Occup Environ Health (in press).

6 Viikari-Juntura E, Riihimäkki H, Tola S, Videman T, Mutanen P. Neck trouble in machine operating, dynamic physical work and sedentary work: a prospective study on occupational and individual risk factors. $\mathcal{F}$ Clin Epidemiol 1994;47:1411-22.

7 De Zwart BCH, Broersen JPJ, Frings-Dresen MHW, Van Dijk FJH. Repeated survey on changes in musculoskeletal complaints relative to age and work demands. Occup Environ Med 1997;54:793-9.

8 Hernberg S. Work-related diseases: some problems in study design. Scand $\mathcal{F}$ Work Environ Health 1984;10:367-72.

Östlin P. The health-related selection effect on occupational morbidity rates. Scand F Soc Med 1989;17:265-70.

10 Olsen J, Sabroe S. Health selection among members of a Danish

1 Dowe GR, Chiarelli AM, Lindsay JP. Components and 1 Howe GR, Chiarelli AM, Lindsay JP. Components and modifiers of the healthy worker effect: evidence from three occupational cohorts and implications for indus

12 Van Dijk FJH, Weel ANH, Broersen JPJ. Identification of work-related diseases by periodic occupational health surveys: experiences in the Netherlands. In: Mehlman MA, Upton A, eds. Advances in modern environmental toxicology. Princeton: Princeton Sci Publ, 1994;23:75-91.

13 De Zwart BCH, Broersen JPJ, Van der Beek AJ, FringsDresen MHW, Van Dijk FJH. Occupational classification according to work demands: an evaluation study. Int $\mathcal{f}$ Occup Med Environ Health (in press).

14 Andersson GBJ. Epidemiologic aspects on low-back pain in industry. Spine 1981;6:53-9.

5 Hosmer DW, Lemeshow S. Applied logistic regression. New York: Wiley, 1989.

16 Van Veldhoven $M$, Van Dijk FJH, ThF Meijman. Hoe periodiek is het periodiek bedrijfsgezondheidkundig onderodiek? (How periodic is the periodic occupational health survey?). Tijdschrift voor Sociale Gezondheidszorg 1993;71. 408-14.

17 Tuomi K, Toikkanen J, Eskelinen L, Backman A-L Ilmarinen J, Järvinen E, Klockars M. Mortality, disability 
and changes in occupation among aging municipal employees. Scand fo Work Eviviron Health 1991:17(supp 1):58-66

18 Arndt V, Rothenbacher D, Brenner H, Fraisse E, Zschenderlein B, Daniel U, et al. Older workers in the conZschenderlein B, Daniel U, et al. Older workers in the construction industry. results of a routine health examination al a five year follow up. Occup Environ Med 1996;53.686Backman A-L, Järvinen E. Turnover of professional drivers Scand f Work Environ Health 1983;9:36-41.

20 Hedberg GE, Langendoen SM. Factors influencing the turnover of Swedish professional drivers. Scand $\mathcal{F}$ Soc Med 1989;17:231-7.

21 Heuer H, Klimmer F, Kylian H, Seeber A, Schmidt K-H Hoffmann G, Luttke-Nymphius M. Musculoskeletal problems in bricklayers as a function of length of employment: the role of secondary selection by low-back pain. Work Stress 1996;10:322-35.
22 Riihimäki H. Back and limb disorders. In: McDonald JC, d. Epidemiology of work related diseases. London: BMJ, 1995, 207-38

23 Koskela R-S. Occupational mortality and morbidity in relation to selective turnover. Scand $\mathcal{F}$ Work Environ Health 1982:8 (suppl 1):34-9.

24 Hildebrandt $\mathrm{VH}$ Back pain in the working population: prevalence rates in Dutch trades and professions. Ergonomprevalence rates in $1995 ; 38: 1283-98$.

25 Kilbom A, Winkel J, Karlquist L. Is physical load at work reduced with increasing age? A pilot study. Book of abstracts: Second International Scientific Conference on Prevention of Work-related Musculoskeletal Disorders; September 1995. Montreal: Institut de recherche en santé et en sécurité du travail du Québec, 1995:60-2.

26 Lavender SA, Marras WS. The use of turnover rate as a passive surveillance indicator for potential low back disorders. Ergonomics 1994;37:971-8.

\section{Occupational and Environmental Medicine and the electronic age}

OEM has an Email address which is 100632.3615@compuserve.com. We welcome contact by Email, including letters to the editor. Some of our reviewers already send us their reports by Email, helping to speed up the peer review process.
Our publishing system is now fully electronic, and authors are sending their revised copy to us on disk as well as paper. Watch for revised Instructions to Authors.

The Editor 